\title{
Quatre siècles et demi de New (New) Law \& Economics: du pragmatisme juridique dans le régime consulaire de contrôle social des marchés ${ }^{1}$
}

Cet article examine les caractéristiques du régime consulaire de contrôle social des marchés en s'appuyant sur une étude du fonctionnement du tribunal de commerce de Paris, une institution créée au $\mathrm{xvl}^{\mathrm{e}}$ siècle. L'examen du raisonnement pragmatique des juges de l'économie et son rapprochement avec le « pragmatisme expérientiel » ou le " pragmatisme quotidien » de Richard Posner, figure emblématique de ce que nous appelons la New (New) Law \& Economics, met au jour des formes de capture institutionnelle et de néo-corporatisme qui incitent à repenser l'action publique à partir d'une critique de ce régime consulaire.

This paper examines the main characteristics of the consular regime of social control of markets by looking at some aspects of the organization and operation of the Commercial Court of Paris, a four and a half century old institution in which judges are lay, business people. Comparing the pragmatic legal reasoning of the consular judges with Richard Posner's « experiential pragmatism » or " everyday pragmatism » points to a combination of old forms of institutional capture and new forms of corporatism that increasingly serve as a model for the management of core State functions. This raises the issue of finding news forms of protection of the public interest in a market society.

Mots clés : pragmatisme juridique $\bullet$ marchés $\bullet$ contrôle social $\bullet$ law $\&$ economics $\bullet$ capture institutionnelle

Key words: legal pragmatism • business • social control • law \& economics • institutional capture

Emmanuel LAZEGA, Université Paris-Dauphine, IRISES/CERSO-CNRS et CMH-CNRS elazega@ens.fr

'Les positions prises dans cet article n'engagent que leur auteur. Ce texte propose une réflexion qui émane d'une recherche empirique réalisée avec Lise Mounier et avec le soutien de la Mission de Recherche "Droit et Justice " du ministère de la Justice, du Programme "Cognitique du ministère de la Recherche, et de I'Institut universitaire de France. Je remercie Sophie Harnay pour ses critiques et suggestions. Cette recherche n'aurait pas vu le jour sans le soutien organisationnel d'Alexis Ferrand, Philippe Rollet, Chantal Figueredo, Sophie Goyat et Sylvie Kleczewski que je remercie de leur aide lors de la réalisation de différentes étapes de l'enquête. Je remercie aussi les rapporteurs anonymes de la RFSE qui m'ont aidé à améliorer ce texte. 


\section{Le modèle consulaire de contrôle social de l'activité économique}

Le contrôle social et la régulation des activités entrepreneuriales et marchandes ont toujours fait l'objet d'une lutte d'influence relativement déséquilibrée entre l'État, le monde des affaires (les entreprises et leurs représentants institutionnels) et les consommateurs. Cette lutte s'intensifie dans une société elle-même organisationnelle et marchande. En sciences sociales, la régulation externe (par l'État) et l'autorégulation des marchés (par le monde des affaires essentiellement) sont habituellement étudiées de façon séparée, ce qui conduit à une simplification abusive dans la compréhension de l'organisation sociale des marchés, et donc du fonctionnement de la société marchande. La notion de « régulation conjointe » - empruntée à Jean-Daniel Reynaud (1989) et étendue à un contexte plus large [Lazega et Mounier, 2003] - et l'étude de ses formes institutionnelles permettent de dépasser cette simplification. Par exemple, cette régulation conjointe peut prendre la forme de professions libérales spécialisées ${ }^{2}$, de la responsive self-regulation étudiée par les Anglo-saxons [Ayres et Braithwaite, 1992]. Un autre exemple de régulation conjointe est fourni en France par un système complexe de coopération entre l'État, les corps intermédiaires locaux et les individus de la société civile, système qui crée des institutions particulières comme les chambres de commerce et les tribunaux consulaires (prud'hommes et tribunaux de commerce). Ces institutions consulaires représentent des manières spécifiques de partager les coûts de la régulation et du contrôle social des marchés, mais aussi de mobiliser l'expérience des parties prenantes, leur connaissance du monde du travail et de l'économie, dans la mise en œuvre de ce contrôle social.

Les solutions institutionnelles connues pour résoudre ce type de problèmes peuvent différer selon les pays, voire au sein d'un même pays. En France, c'est en partie le rôle des tribunaux de commerce de résoudre les conflits entre acteurs économiques et d'exercer une certaine discipline sur l'entrée, l'activité et la sortie des marchés (contentieux commercial et faillites). Les données relatives à ces juridictions spéciales pour le commerce sont particulièrement pertinentes pour l'étude de cette régulation conjointe car cette institution consulaire existe depuis quatre siècles et demi. Elle représente une des seules institutions qui ont traversé presque inchangées la Révolution de 1789 , tout en ayant été régulièrement - et même depuis ses débuts remise en cause de façon fondamentale. Elle est le parfait exemple d'une institution intermédiaire dont l'existence est difficile à justifier en théorie (juridique et politique classiques), mais qui s'est toujours imposée dans la pratique, à la fois en vertu d'une demande (d'une partie) des intéressés et de l'État.

L'État y partage depuis très longtemps son propre pouvoir judiciaire avec la communauté d'affaires locale. Ce sont des juges consulaires qui y siègent. Ces juges, qui se disent volontiers représentants de la "société civile économique ", ne sont pas rémunérés. Ils sont élus pour un mandat de deux ou de quatre années (pour une durée totale maximale de quatorze ans) par un corps électoral composé des juges

${ }^{2}$ Voir par exemple le cas des avocats d'affaires [Lazega, 1995, 2003], mais aussi celui des experts comptables et des notaires. 
déjà en place et des délégués des syndicats patronaux à la Chambre de commerce de leur localité, après avoir été parrainés par un syndicat patronal et, par exemple à Paris, présélectionnés par un Comité émanant de cette même Chambre de commerce. En théorie, les deux institutions économiques consulaires (la Chambre de commerce et le tribunal de commerce) s'appuient mutuellement dans l'exercice de cette forme de contrôle social des marchés et entretiennent des liens étroits ${ }^{3}$. Dans cette solution institutionnelle, les coûts du contrôle sont partagés par l'État, les industries ou les entreprises et les juges individuels. En théorie cependant, chaque juge agit comme juge individuel, sans mandat spécifique d'un secteur de l'économie.

La valorisation particulière, dans la mise en œuvre de ce contrôle social, de l'expérience des juges du commerce et de leur connaissance du monde de l'économie, s'accompagne, chez eux, de la revendication d'un certain pragmatisme dans la prise de décision judiciaire. Cet article rapproche cette revendication des théories de Richard Posner (1993 et 1995) - que nous appelons New (New) Law \& Economics (N(N)LE) - sur le «pragmatisme expérientiel » ou le « pragmatisme quotidien » des juges. II fait apparaître l'existence, sous-jacente au pragmatisme juridique « individuel » enraciné dans l'expérience quotidienne du juge, d'un pragmatisme juridique « collectif » porteur de valeurs et d'intérêts néo-corporatistes. Ce constat soulève la question politique, audelà des formes de pragmatisme dans la mise en œuvre du contrôle social du monde des affaires, d'une redéfinition du rôle et de la structure des corps intermédiaires, et de l'extension du modèle consulaire dans les institutions semi-privées ou autorités administratives indépendantes de plus en plus nombreuses auxquelles l'État délègue ses pouvoirs. En ce sens, il invite la sociologie politique à repenser l'action publique à partir d'une critique du régime consulaire.

\section{Une institution pragmatique de régulation conjointe de l'économie}

En France, ces litiges privés sont traités par des tribunaux de différentes natures, localisés dans différentes villes avec un ressort géographique défini ${ }^{4}$. L'article L.411-1 du Code de l'organisation judiciaire définit les tribunaux de commerce comme des juridictions spécialisées du premier degré dont la compétence spécifique, fixée par le Nouveau Code de procédure civile et par le Code de commerce, est de trancher les litiges commerciaux entre commerçants, personnes physiques et morales, c'està-dire entre entreprises, mais aussi par exemple entre une entreprise et un consommateur individuel engagés dans un acte commercial. Les articles 631 et suivants du Code de commerce déterminent les litiges qui doivent être portés devant le tribunal de commerce, à moins que les parties conviennent par avance - au moyen d'une clause compromissoire - de soumettre leurs litiges à des arbitres. Très généralement, les principales sortes de contestation entrant dans la compétence du tribunal de commerce sont relatives aux engagements et transactions entre commerçants, aux actes de commerce entre toutes personnes et des contestations entre associés pour raison

\footnotetext{
${ }_{3}^{3}$ Pour une contextualisation historique de cette institution intermédiaire, voir par exemple Hilaire (1999), Hirsch (1985), Ithurbide (1970), Lafon (1981), Lemercier (2002) ; sur la construction d'une identité de juge non statutaire, voir Lazega et Mounier (2008a et 2008b).

${ }^{4}$ Rappelons cependant qu'un différend peut se résoudre autrement que par un jugement. Les parties en litige peuvent recourir à la conciliation, à la médiation judiciaire, à l'arbitrage, à la composition amiable, à la transaction.
} 
d'une société de commerce [Coutant, 1988]. De plus, les tribunaux de commerce sont compétents en matière de procédure collective (redressement ou liquidation) et, plus récemment, en matière de prévention des difficultés des entreprises. Certaines restrictions à la compétence des tribunaux de commerce portent sur les litiges relatifs aux accidents du travail, aux baux commerciaux, aux marques de fabrique et certains brevets ${ }^{5}$. Les litiges traités ont souvent un caractère trivial car ce sont essentiellement des contentieux contractuels et concernant des recouvrements de créances qui dominent le rôle des tribunaux de commerce en France, probablement le contentieux commercial dans la plupart des pays. Le travail des juges du commerce peut néanmoins devenir très vite très complexe, même fortement défini et encadré par la procédure, car marqué par les conflits de normes, souvent par la « mauvaise foi » des parties.

La loi du 16 juillet 1987 dispose que les juges sont élus par un collège électoral comprenant les délégués consulaires ${ }^{6}$, les membres en exercice des tribunaux de commerce et des chambres de commerce, des anciens membres des chambres de commerce et des tribunaux de commerce qui en ont fait la demande. La liste électorale est établie par une commission présidée par un juge commis à la surveillance du registre du commerce et des sociétés. Les juges doivent être âgés de trente ans au moins, être inscrits sur les listes électorales du ressort du tribunal de commerce ainsi que sur le registre du commerce et des sociétés depuis au moins cinq ans au ou avoir exercé pendant cinq ans une fonction de direction (PDG, administrateur général, etc.). Les causes d'inéligibilité sont le fait d'avoir soi-même subi une faillite ou d'avoir été juge du tribunal de commerce pendant quatorze ans de manière continue. La fonction de juge au tribunal de commerce est bénévole, au sens où elle n'est pas rémunérée par l'État : le juge consulaire peut être à la fois magistrat au tribunal et rémunéré par l'entreprise qui l'emploie à l'extérieur du tribunal. Le premier mandat est d'une durée de deux ans, les suivants de quatre ans. Les juges consulaires ont les mêmes pouvoirs et prérogatives que les juges de carrière, magistrats de l'ordre judiciaire. Ills sont aussi soumis aux règles d'abstention et de récusation du Nouveau Code de procédure civile (articles 339 à 341).

Un tel régime de régulation conjointe est justifié de plusieurs manières. Premièrement, c'est une forme de justice moins dispendieuse et plus rapide qu'un système qui fait appel à des juges de carrière. Le monde des affaires endosse une plus grande partie des coûts du règlement de ses propres conflits et les retards et les attentes y sont moins longs que dans les tribunaux traditionnels de grande instance. La rapidité est en effet une valeur essentielle du régime consulaire ${ }^{7}$. Deuxièmement, les juges

\footnotetext{
${ }^{5}$ Peu d'informations systématiques sont disponibles au niveau national sur les tribunaux de commerce, sur leur travail et sur le genre d'affaires traitées. Les données statistiques désagrégées sont inaccessibles car propriété du Greffe, une société privée dont le statut remonte à l'Ancien Régime. Les statistiques officielles de chaque tribunal concernent essentiellement le nombre de cas traités en matière de contentieux et de faillites, ainsi que la mesure du temps de traitement. Une exception notable se trouve dans le travail statistique de Haehl et al. $(1992,1993)$ resté malheureusement sans extension.

${ }^{6}$ Les délégués consulaires sont les correspondants économiques de Chambre de commerce et d'industrie pour les circonscriptions auxquelles ils appartiennent. Leurs listes électorales sont dressées par catégorie et sous-catégorie professionnelle. Les électeurs comprennent, entre autres, les commerçants immatriculés au registre du commerce de la circonscription de la Chambre de commerce, les chefs d'entreprise inscrits au répertoire des métiers et immatriculés au même registre du commerce, etc.

${ }^{7}$ Les effets pervers de la rapidité sont difficilement mesurables sans accès aux statistiques désagrégées des procès. De plus, cette valeur est d'autant plus promue par le régime consulaire que le tribunal est instrumentalisé par les entreprises pour renégocier leurs contrats (autant que pour accéder à la justice).
} 
de carrière - qui sont des fonctionnaires - sont souvent considérés comme inexpérimentés, peu formés à comprendre les problèmes des entreprises ou capables de contrôler de façon appropriée le comportement des dirigeants d'entreprises, particulièrement en matière d'insolvabilité et de faillite [Carruthers et Halliday, 1998, p. 431]. Troisièmement, le droit des affaires ignore souvent les us et coutumes idiosyncrasiques désignés comme des « usages » dans les tribunaux de commerce, basés sur les cultures traditionnelles particulières de l'industrie ou du commerce et caractérisant le secteur entier. Le règlement efficace des conflits ne peut passer outre l'ensemble des règlements et des conventions [Favereau, 1994] qui structure différemment les pratiques du monde des affaires dans chaque secteur traditionnel. Les juges du tribunal de commerce, qui sont supposés être des hommes et des femmes d'affaires expérimentés, sont considérés comme des spécialistes de leur domaine professionnel et sont par conséquent dans une meilleure position (que des fonctionnaires permanents) pour en connaître les coutumes, les adapter rapidement au milieu instable ou changeant des affaires et aussi pour innover en matière de réglementation.

Le tribunal de commerce français a donc des caractéristiques spécifiques qui donnent une visibilité accrue à certains aspects du lien entre les mécanismes juridiques (exogènes) et sociaux (endogènes) de la régulation dans le domaine du commerce. L'État ne sanctionne pas tout seul. II requiert la participation et les investissements d'intervenants - tant des particuliers que des entreprises - qui sont prêts à à assumer une partie des coûts du contrôle. De fait, les juges élus accomplissent ce qui est habituellement considéré comme une fonction régalienne de l'État. Ce cas assez particulier de régulation conjointe pousse à l'extrême la « co-régulation » [Grabosky et Braithwaite, 1986, p. 83], entendue comme une autorégulation par des associations industrielles qui sont reconnues par l'État. Pour les juges du tribunal de commerce de Paris, cependant, rappeler les usages ne signifie pas représenter des intérêts corporatistes. Les médias, le public et les commissions d'enquête [Montebourg et Colcombet, 1998] soupçonnent souvent que la cooptation dans les nominations avait pour résultat l'élection de juges qui ne parviendraient pas, par la suite, à prendre des distances par rapport à leur milieu d'origine, par exemple le secteur qui avait parrainé leur candidature, et donc d'intérêts particuliers. Les parties n'ont souvent qu'une confiance limitée en l'impartialité des décisions du tribunal, particulièrement dans les tribunaux de commerce des petites villes. Ils craignent que les juges de ces petits tribunaux puissent exercer un contrôle judiciaire sur leurs propres concurrents ${ }^{8}$.

Généralement, le monde des affaires a certaines attentes vis-à-vis des tribunaux de commerce modernes : la rapidité et la fermeté, peu d'appels, une séparation nette

${ }^{8}$ Ce déficit de légitimité se traduit par le serpent de mer des tentatives de réforme des juridictions commerciales. Le système de justice commerciale français évolue aussi, avec l'État qui a tenté d'accroître sa présence au tribunal de commerce. Depuis 1981, le procureur général a un bureau au tribunal et peut participer au délibéré des juges, en particulier dans les cas de faillites (qui se jugent à huis clos et peuvent avoir une dimension pénale et / ou fortement sociale). En 2001 et en 2002, les tentatives socialistes pour convaincre l'Assemblée nationale et le Sénat d'introduire des juges professionnels permanents parmi les juges consulaires ont échoué. Les réformateurs présentaient cette " mixité » comme un moyen de rassurer les commerçants impliqués dans des poursuites judiciaires en améliorant le contrôle sur les contrôleurs. Il est intéressant de souligner que cette réforme entraînait de fait une situation paradoxale: du temps où l'État français était plus directement actif qu'il ne l'est aujourd'hui dans le monde des affaires, la justice commerciale fonctionnait sans réelle supervision de la part de la Chancellerie. Depuis les années 1980, en revanche, alors que l'État a commencé à se retirer du contrôle direct de l'économie, il a parallèlement entrepris d'accroître sa présence dans les tribunaux de commerce et, par conséquent, son contrôle du processus judiciaire. Pour justifier ce changement de politique, l'État affirme essentiellement vouloir rassurer les investisseurs européens et internationaux qui demandent un traitement équitable dans les tribunaux de commerce consulaires français. 
entre le patronage personnel et la prise de décisions judiciaires, ainsi que le plus de neutralité possible. Dans le cas des tribunaux consulaires, des secteurs entiers du monde des affaires, représentés par leurs syndicats patronaux, ont intérêt à être représentés au tribunal notamment pour défendre les coutumes liées à leur activité ou à leur profession [Carruthers et Halliday, 1998, p. 488]. Ainsi, du point de vue de certains secteurs de l'économie, les juges consulaires sont plus que de simples juges. Ce sont des entrepreneurs judiciaires [McIntosh et Cates, 1997] qui représentent la sensibilité du syndicat patronal qui les a initialement aidés à siéger au tribunal en parrainant leur candidature. Les juges eux-mêmes ne partagent pas tous cet avis. Lors de nos entretiens, ils déclarent officiellement qu'une fois nommés ils sont indépendants, qu'ils n'ont aucun mandat de leur secteur d'origine et se disent totalement impartiaux. II n'en demeure pas moins qu'ils sont consultés sur une profession ou une activité particulière. Ainsi, en pratique, il est difficile de dissocier totalement représentativité et compétences. Le système n'en représente pas moins un régime typique de régulation conjointe où les institutions, le monde des affaires et les particuliers acceptent de partager ensemble les coûts du contrôle.

Le tribunal de commerce de Paris a une spécificité par rapport à la plupart des autres tribunaux de commerce. Sa taille est plus importante, le nombre des chambres spécialisées ${ }^{9}$ plus élevé ainsi que le nombre d'affaires traitées. À lui seul, le tribunal de commerce de Paris traite 10 à $12 \%$ de l'ensemble du contentieux commercial traité en France (arbitrage non compris). En 2002, il a rendu 101201 décisions ${ }^{10}$. Il est différent également par la diversité des secteurs de l'économie représentés par ses juges, par l'importance de la clientèle, puisque la demande et la défense des sociétés se fait à leur siège social - cette dernière caractéristique étant partagée avec les tribunaux de Nanterre, Bobigny, Créteil et ceux des grandes villes de province. La formation initiale des juges est souvent d'un haut niveau ( $X, E N A, H E C$, Sciences-Po, doctorat en droit), mais seule la moitié d'entre eux a une formation juridique. La multiplicité de leurs expériences des affaires dans de nombreux domaines forme une sorte de « capital de compétences » que le tribunal de commerce de Paris cherche à exploiter par ce que nous appellerons plus loin une « culture de la consultation ». La plupart ne sont plus des commerçants ou entrepreneurs au sens traditionnel du terme, mais des cadres ou d'anciens cadres supérieurs de grandes entreprises (un juge dira : «c'est l'ère des managers »).

Notre recherche empirique a consisté en trois vagues d'entretiens approfondis auprès de l'ensemble des juges consulaires du tribunal de commerce de Paris ${ }^{11}$. Ces entretiens ont permis, dans une certaine mesure, d'évaluer cette forme de légitimité transversale construite par la magistrature consulaire contemporaine.

\footnotetext{
${ }^{9}$ Droit des sociétés, Droit international, Droit communautaire, Multimédia et nouvelles technologies, Fonds de commerce, Concurrence déloyale et contrefaçon.

${ }^{10} 41229$ jugements rendus au fond, 7425 ordonnances de référés, 19687 ordonnances de juges-commissaires, 12907 ordonnances d'injonction à payer, 4715 jugements d'ouverture de procédures collectives, 7298 autres jugements de procédures collectives et 8940 ordonnances sur requêtes (Les chiffres clés 2002 du tribunal de commerce de Paris).

${ }^{11}$ Cette étude du tribunal de commerce de Paris a été réalisée, pour la première vague à l'automne 2000, à partir d'une liste de 157 juges, présents au tableau des audiences du second semestre 2000 auxquels nous avons ajouté 10 personnes - les sages et les responsables des associations. Pour la seconde vague de l'enquête, réalisée en 2002, nous avons établi une liste de 197 juges. Cette liste réunit les juges présents sur la liste de la première vague (encore en activité au tribunal ou ayant quitté le tribunal) auxquels il faut ajouter les élus à l'automne 2000 et 2001. La troisième vague d'entretiens a eu lieu à l'automne 2005 ; une liste de 234 juges a réuni les présents du tableau 2005 (166 juges) et les présents des vagues précédentes ainsi que les nouveaux élus depuis 2002. Les rapports de recherche sont disponibles auprès de la Mission de recherche Droit \& Justice du ministère de la Justice [Lazega, Mounier et al., 2001, 2003, 2007].
} 


\section{Pragmatisme, usages et jugement en équité}

Du point de vue des notions de litige, de parties, de respect du contradictoire et de l'obligation de juger en droit (art. 12 du NCPC), la justice consulaire n'est pas, en théorie, dérogatoire au droit commun. Néanmoins, quelques éléments de procédure commerciale " pratique », telle que nous l'avons observée ethnographiquement et telle que les avocats d'affaires expérimentés l'enseignent à l'École de formation du Barreau de Paris permettent de définir le contexte dans lequel le raisonnement pragmatique prend une partie de son sens. Les juges consulaires raisonnent beaucoup en utilisant des précédents, mais la jurisprudence est opaque (comme dans toutes les juridictions de premier degré). Très souvent, le contentieux commercial n'est pas prioritairement une affaire de justice, mais un moyen de renégociation des contrats instrumentalisant le tribunal. Les juges n'aiment donc pas beaucoup que les avocats assignent sans tentative, au préalable, d'un règlement amiable. Les magistrats consulaires se sentent proches des justiciables : ils apprécient que les parties se déplacent (surtout pour la plaidoirie) ; ils aiment leur poser des questions dans un langage non juridique. Au commerce, les avocats ont souvent, de ce fait, une peur bleue des réactions incontrôlées de leur client dans ces interactions. Les juges cherchent des alternatives : qu'on leur présente des demandes subsidiaires permettant d'aider les parties à transiger, donner raison aux uns et aux autres, expliquer aux perdants pourquoi ils ont perdu.

L'utilisation de précédents par des juges les rapproche, dans une certaine mesure, d'un fonctionnement de type Common Law. Dans ce contexte, en pratique, le pragmatisme est revendiqué par le juge comme l'évaluation des effets de sa décision sur le fonctionnement de l'économie : comptent avant tout les conséquences que les règles de droit, les précédents, les décisions et les institutions ont sur le monde des faits. Ici, une décision est pragmatique en ceci qu'elle procède de l'adjonction de connaissances d'économie et de gestion aux connaissances du droit et de jurisprudence dans la prise de décision judiciaire. En pratique cela se traduit par jugement qui produit sa propre synthèse de droit, de gestion et d'économie, mais s'appuyant sur les usages du monde des affaires, sur le bon sens et sur l'équité.

Notre propos ici n'est pas de faire la part du droit positif et des autres critères de décision sur lesquels les juges consulaires s'appuient. À supposer que ce travail soit possible, il est rendu impossible pour l'instant en raison de l'inaccessibilité des statistiques des procès. II reste que les juges consulaires se réservent ouvertement la possibilité, au nom de leur pragmatisme et de leur expérience, de pratiquer cette synthèse. Deux exemples de composantes non juridiques interviennent dans cette dernière : l'équité et les usages.

Le jugement en équité est mis en avant par beaucoup de juges consulaires lorsqu'ils parlent de leur travail non routinier. La prise en compte, dans le délibéré, de l'expérience et des usages, va de pair avec des jugements en équité. II est intéressant de relever que le poids relatif du jugement en équité par rapport au jugement en droit semble varier d'un juge à l'autre. Pour les uns, on doit évidemment apprendre à ne pas juger en équité. L'équité présuppose des raisonnements qui donnent prise à des influences extérieures diminuant l'indépendance. Pour les autres, c'est précisément 
la spécificité du tribunal de commerce que de permettre de tenir compte de l'équité, même si elle pose des problèmes de définition. Dans le travail du juge pragmatique, il faut trouver les décisions et règles qui produisent les meilleures conséquences. Ces dernières peuvent être à court ou long terme, systématiques ou singulières. Rendre le droit pragmatique peut signifier toutes sortes de choses et l'efficacité peut avoir toutes sortes de significations. Ce qui compte est que le critère soit pratique, que son contenu spécifique soit malléable.

Les citations suivantes, extraites des entretiens que nous avons eus avec les juges du TCP, illustrent l'importance du raisonnement en équité pour beaucoup d'entre eux :

« Vous avez les tribunaux de l'ordre civil qui sont des professionnels du droit qui appliquent le droit stricto sensu, nous, on doit voir le droit et les incidences économiques que ça a. Alors bien sûr, on ne peut pas avoir de jugements qui soient contraires totalement à la loi, mais on doit trouver un moyen terme... On est juge, mais juge du commerce et on doit avoir une vue plus économique que purement juridique. » (Juge $n^{\circ} 72$ )

« [...] La justice doit être rendue, et on rend justice en droit. On essaye de la rendre en droit et en équité, mais c'est pas toujours facile. Il est évident que c'est au tribunal de commerce que vous avez le plus de chances d'avoir une justice rendue en droit et en équité. Et je crois que ça c'est un point qui tend à se perdre de plus en plus, et avec la réforme dont on parle, on séloigne de beaucoup des raisons pour lesquelles les Tribunaux de commerce ont été créés ». (Juge n 101, en 2000)

«Une des qualités très importantes, c'est d'avoir du bon sens, peut-être que c'est la première qualité. II faut un minimum de compétences -si on n'est pas juriste on les acquiert- mais il faut du bon sens, et de l'impartialité. II faut oublier un peu l'équité, parce que c'est une tendance naturelle de juger en équité. Il y a un article du Code qui le permet, quand on ne sait plus quoi faire. Mais souvent on a une opinion en équité, et on cherche si possible à la justifier, sinon tant pis. Parfois on commence à rédiger un jugement et on change les conclusions, parce que le raisonnement ne passe pas. J'insiste, plus que des qualités juridiques, il en faut, il faut un certain esprit d'analyse et de synthèse, pour simplifier dans le fatras des dossiers des avocats, une fois j'en ai eu un haut comme la table. II faut des qualités juridiques, mais en fait souvent il ne faut pas trouver des arguments juridiques, mais arbitrer entre ceux des parties. » (Juge $n^{\circ} 97$ )

" Je pense qu'il faut connaître le commerce pour sentir où est le vrai litige, pas celui qu'on nous annonce. (...) Je pense que pour être bien dans les circonstances qu'on vous présente, d'un litige, il faut avoir vécu le commerce intensément. Et à tous les niveaux. Pas l'avoir vécu au niveau PDG. II faut l'avoir vécu, il faut avoir eu une vie de commerce. Et c'est la force du TC, c'est d'avoir en son sein des gens qui sont des commerçants. » (Juge $n^{\circ} 158$ )

II faudrait évidemment plus que les affirmations des interviewés pour conclure que les juges consulaires statuent systématiquement en équité [Hilaire, 1998], surtout pour les litiges triviaux qui n'exigent qu'une simple distribution de titres exécutoires. On peut penser qu'un problème de facture impayée peut être considéré comme trivial, ne soulevant aucun problème juridique et ne donnant aucune marge de 
manoeuvre au juge qui doit trancher en droit. Reste que même un simple problème de facture impayée peut appeler un travail complexe d'interprétation. Elle peut renvoyer à d'autres problèmes auxquels les juges pragmatiques ne sont pas insensibles, déclenchant une lecture de l'affaire moins attachée au droit. Un avocat raconte avec admiration le travail d'un président de chambre en 2005 :

"Une société faisait payer des abonnements de télésurveillance sur 3-4 ans. Une PME qui louait ces services les payait par trimestre. Elle oublie de payer. La société de télésurveillance fait le sous-marin pendant un, deux, trois, quatre loyers, puis envoie une mise en demeure : « Payez les quatre loyers, sinon résiliation avec assignation au tribunal de commerce ». Au tribunal, elle demande de condamner la petite SARL à payer tous les loyers sur la durée de tout le contrat. Dans ce genre de cas, c'est David contre Goliath. La société de télésurveillance revient souvent avec les mêmes assignations. J'ai dû la voir cinq fois en quatre mois. Les Goliath ont gain de cause parce qu'elles ont blindé leur contrat. Surtout au commerce où c'est assez simple. C'est l'application d'un contrat par deux sociétés. Point. « Ce sont deux professionnels, ils ont signé ». Même si la SARL est une petite PME. La réflexion consumériste sur la protection du consommateur n'a pas encore atteint le tribunal de commerce. Mais le Président, ça le gonflait. II leur disait « Je vous vois tout le temps avec la même rengaine, les mêmes excuses pour faire courir le loyer et matraquer derrière ». II a introduit un raisonnement spécial via la modération de la clause pénale : le juge vient modérer les pénalités initialement prévues contre la partie qui ne paie pas son loyer. Un juge professionnel peut le faire en suivant une jurisprudence. Mais ici, il a trouvé un argument juridique contre une solution standard qui le choquait. Le juge du commerce a d'abord sa solution en tête et ensuite il rédige son jugement. Le fameux syllogisme est trouvé après! Je ne trouve pas ça choquant. »

Dans l'esprit des juges, le jugement pragmatique renvoie souvent à des « sensibilités » et des expériences individuelles. Cependant, un second exemple, celui des us et coutumes idiosyncrasiques désignés, dans les Tribunaux de Commerce, comme des « usages », montre que le pragmatisme n'est pas seulement une caractéristique individuelle. Il est aussi associé à des normes plus collectives du monde des affaires. L'expression consacrée «Les usages sont sources de droit » est si souvent évoquée par les juges qu'elle pourrait être inscrite au fronton du tribunal de commerce. Le droit des affaires ignore souvent ces usages basés sur les sous-cultures traditionnelles de l'industrie ou du commerce. En particulier en matière de contrats, ces usages commerciaux guident aussi les opérations commerciales et sont reconnus par le tribunal de commerce.

Le Guide pratique du magistrat consulaire (2001 : 134) définit un usage comme « une pratique qui, pour une profession et une région déterminée, a force obligatoire dans les contrats ». Il y a des usages locaux (par exemple, usages des ports de commerce pour la réception et la délivrance des marchandises expédiées par mer), des usages nationaux et des usages internationaux (par exemple, conditions générales de vente des fonderies européennes). Certains de ces usages ont été incorporés à la loi (par exemple, usages commerciaux sur les ventes commerciales), d'autres sont incorporés dans des contrats type (par exemple, la règle d'York et d'Anvers sur les avaries communes en transports maritimes). Si un usage commercial ne peut être contraire à une 
loi commerciale impérative, il peut par contre déroger aux règles du Code civil qui ne sont pas d'ordre public (par exemple, présomption de solidarité dans les opérations commerciales et capitalisation trimestrielle des intérêts en matière de comptes courants bancaires). S'il n'est pas de notoriété publique, l'usage doit être prouvé par celui qui l'invoque. Cette preuve peut prendre la forme d'une attestation délivrée par la chambre de commerce et d'industrie ou par un organisme professionnel faisant autorité comme l'Association française des banques $»^{12}$.

Les usages au sens ainsi défini sont des normes sectorielles et des spécifications techniques reconnues et déposées au greffe du tribunal. Même si l'activité commerciale est aujourd'hui fortement formalisée dans des contrats écrits et que le recours à ces normes reconnues reste exceptionnel, le recours à des normes externes au droit positif est légitime et valorisé au TCP. Ces normes et spécifications reconnues n'épuisent pas les conventions et critères non juridiques auxquels les magistrats consulaires ont recours pour prendre des décisions de justice, notamment lorsqu'ils disposent d'un fort pouvoir d'appréciation.

\section{Rapprochement du pragmatisme consulaire avec le pragmatisme juridique posnérien}

L'un des arguments principaux légitimant cette institution consulaire est ainsi le pragmatisme basé sur un mélange de compétences et d'expériences acquises par le juge non statutaire, ou profane, au cours de sa carrière d'entrepreneur ou de cadre supérieur d'entreprise. Il est intéressant de remarquer que cette forme de pragmatisme et ses implications soulèvent des questions traitées dans des théories et des débats actuels dans le monde juridique anglo-saxon. Nous pensons que le fait de considérer le tribunal de commerce comme une illustration particulièrement intéressante de ce pragmatisme anglo-saxon (plutôt américain) éclaire certains aspects de cette institution consulaire et rend explicites certaines implications de son fonctionnement.

Le pragmatisme juridique anglo-saxon, en particulier américain, est une théorie critique de la conception traditionnelle du droit et de la prise de décision judiciaire [Brint et Weaver, 1991]. En particulier dans les pays de la Common Law, la représentation classique du droit s'appuie sur une jurisprudence, une analyse de faits juridiques universels et abstraits, des précédents et une argumentation rigoureuse utilisant l'analogie dans des controverses purement juridiques. Le pragmatisme juridique, pour sa part, considère cette approche comme trop légaliste, naïvement rationaliste et basée sur une méconnaissance du fonctionnement pratique des institutions judiciaires. II insiste sur la nécessité de prendre en compte d'autres données que purement juridiques (en raison d'un certain éclectisme du droit provenant de ses objectifs multiples), sur le fait que le droit est une pratique instrumentale, située dans un contexte économique, politique et social sans fondements abstraits et universels sûrs, toujours rattachée à une, voire plusieurs perspectives différentes. Le travail de jurisprudence serait caractérisé par ce pragmatisme. Dans ces conditions, le juge n'est pas non plus un

${ }^{12}$ Cette attestation est dénommée "parère ». Depuis 1982, on trouve au greffe du tribunal de commerce de Paris (service des expertises), un bureau de dépôt des usages professionnels, auprès duquel les professions ont été appelées à déposer leurs usages et règlements : il en existait 275 au 31 octobre 2000. 
être purement rationnel capable de reconnaître à l'avance toutes les conséquences de ses décisions. II mobilise ses capacités et sensibilités pour prendre ses décisions de justice. La décision judiciaire apparaît dès lors comme ancrée dans l'expérience du juge, dans son attention au contexte sans fausses croyances a priori en de quelconques fondements universels, dans une jurisprudence empirique et dans la méthode expérimentale appliquée à des contextes complexes et incertains.

D'une certaine manière, personne n'a mieux présenté le pragmatisme dont se réclament les juges du commerce en France que Richard Posner, juge et professeur à I'université de Chicago, mais aussi personnalité complexe [Harnay et Marciano, 2003 ; Krecké, 2003 ; Krecké et Krecké, 2006] ${ }^{13}$. Dans une première période, figure de proue du mouvement de la New Law \& Economics (NL\&E) ${ }^{14}$, il prône une version radicale de cette école [Posner, 1977, 1980] pour laquelle la science économique (néo-classique) doit dire le droit. La maximisation de l'utilité devient synonyme de justice. Les mesures économétriques de l'efficacité remplacent les principes juridiques traditionnels qui servent de fondement à la décision du juge. Le jugement est identifié et réduit à une forme de calcul, cadré par des formules restrictives pour résoudre des conflits juridiques. Le droit doit être rapproché des exigences de l'économie telles qu'elles sont définies par les économistes néo-classiques. L'économie devrait dire le droit.

Cependant, au cours des années 1990, Posner est passé d'un positivisme radical à un pragmatisme plus « mou » et c'est le second Posner que nous utilisons comme point de repère. Nous qualifions de New (New) Law \& Economics [N(N)L\&E] le revirement vers le pragmatisme réalisé par Richard Posner dans certains de ses ouvrages des années 1990. Nous appellerons, de manière quelque peu caricaturale, Posner I le Posner de la première période (positiviste néo-classique) pour le distinguer du Posner de la deuxième période, celle de Problems of Jurisprudence (1993) et de Overcoming Law (1995), que nous appellerons Posner II. Cette distinction Posner I / Posner II n'est pas "homologuée » ${ }^{15}$. Ce revirement (égarement, diraient certains) est largement contesté par l'analyse économique du droit aujourd'hui, y compris parfois par Posner lui-même, un Posner III en quelque sorte. II ne s'agit pas pour nous de considérer que la N(N)L\&E est le début d'un nouveau courant ; il s'agit simplement, à notre sens, d'un moment de vérité dans la compréhension de l'analyse économique du droit telle qu'elle est appliquée dans les tribunaux comme le tribunal de commerce de Paris.

Richard Posner s'inscrit ainsi dans une tradition, riche et hétérogène, de pragmatistes américains (dont les plus récents sont Daniel Farber (1995), Thomas Grey $(1989,1996)$,

\footnotetext{
${ }_{13}$ Pour cette présentation trop rapide du pragmatisme, des débuts et de l'évolution de Richard Posner et de sa conception des juges, je m'inspire directement de Harnay et Marciano (2003), Krecké (2003; Krecké et Krecké, 2006) et Mercuro et Medema (2006). La lecture de ces textes a guidé mon exploration de cette littérature.

${ }^{14}$ Le courant Law \& Economics est apparu à la fin des années 1950 avec le Journal of Law and Economics à l'université de Chicago. Ce début de la littérature économique du droit (généralement appelé "old law and economics ») s'intéressait essentiellement à des domaines du droit explicitement liés à des problématiques économiques (concurrence, concentration industrielle, fiscalité, propriété industrielle). Ce qu'on appelle dans les manuels de base le "new law and economics» (extension de la logique économique à d'autres domaines juridiques) débute avec la publication du livre Economic analysis of law de Richard Posner en 1972.

${ }_{15}$ Posner Il avait plusieurs raisons pour écrire Overcoming Law, notamment la défense de la Common Law contre la tendance à la réglementation légale et à la codification de la législation continentale en Europe. Je me centre sur sa critique du positivisme néo-classique (son autocritique, en quelque sorte) qui m'intéresse davantage. Par ailleurs, signalons que Posner II n'est pas, dans le cadre de cet article, le fils de Richard Posner, Eric, qui publie aussi dans le domaine de l'économie du droit.
} 
Margaret Radin (1990), etc.) dont il n'est pas utile ici de reconstituer la généalogie philosophique (Charles Peirce, William James, John Dewey ; et plus récemment Richard Rorty) et juridique (Holmes, Brandeis, etc.) ${ }^{16}$. Dans ses ouvrages de 1993 et 1995, il insiste sur la primauté des conséquences dans l'interprétation (" the primacy of consequences in interpretation »1995, p. 252). Le critère principal reste l'évaluation par le juge des effets de sa décision sur le fonctionnement de l'économie : comptent avant tout les conséquences que les règles de droit, les décisions et les institutions ont sur le monde des faits (et non plus la mesure étroite de l'efficacité au sens économétrique).

Posner II regrette que l'analyse économique du droit n'ait quitté le formalisme juridique que pour s'appuyer sur des principes tout aussi formels et artificiels proposés par la science économique néo-classique, s'appuyant sur des calculs mathématiques et évitant le monde de l'expérience autant que le faisait le formalisme juridique, finissant par ne plus être des instruments accessibles pour le juge et l'avocat, ne les guidant plus dans leurs décisions quotidiennes. Cette approche positiviste ne parvient pas à s'imposer chez les juges. Sur le plan théorique, elle est aussi fortement critiquée [Sunstein, 2000]. Posner II a ainsi déplacé le fondement de la décision de justice " pragmatique » vers le juge comme personne et vers son expérience du monde au quotidien, une forme d'« pragmatisme expérimentaliste » juridique qui s'appuie sur le pragmatisme américain plus général du début du xxe siècle (John Dewey)[Ogus, 2006]. Posner II reste partisan d'une théorie du droit qui peut servir de cadre pour le juge qui prend des décisions de justice et met en œuvre le droit. II n'a pas renoncé à l'idée d'une jurisprudence positive (Krecké, 2003). Mais la décision de justice n'est plus basée sur la logique (scientifique ou juridique), plutôt sur l'expérience du juge.

Pour Posner II (2003), le bon raisonnement du juge ne peut en effet qu'être subjectif, tacite, basé sur des convictions dérivées de routines ou d'expériences personnelles (sagesse pratique) acquise au cours de la carrière. Le raisonnement pragmatique exige en effet une capacité d'adaptation à des matériaux toujours nouveaux (juridiques ou non, par exemple économiques), une capacité que la connaissance et de la familiarité avec le droit ne développe pas en elle-même, et qui semble requérir un mélange de jugement et d'intuition, tout comme de la prudence, du détachement, de l'imagination et du bon sens. Le raisonnement du juge ne s'appuie plus sur la science économique positiviste. Overcoming law affirme que le pragmatisme est la meilleure théorie normative aussi bien que positive du rôle judiciaire. Le pragmatisme rejette l'idée que le droit est ancré dans des principes fixés et permanents, et réalisé dans des opérations logiques basées sur ces principes, pour affirmer que le droit doit être utilisé comme instrument à des fins sociales. Le pragmatisme est plus une attitude de bon sens et de sens quotidien du raisonnable qu'un corps de doctrines.

Juger, pour Posner Il, suppose à la fois de la logique (comme lorsqu'il se réfère à l'économie) et du sens commun (y compris les normes, coutumes ou croyances). La science économique n'est qu'une source de l'apprentissage juridique parmi d'autres plutôt que l'instrument normatif dans le champ du droit. Il rejette l'idée d'un système explicatif clos et complet, comme prétendait l'être l'analyse économique du droit des débuts de la NL\&E. Même si un voile pudique est jeté sur le critère utilitariste de maximisation

${ }^{16}$ Voir aussi à ce sujet Dickstein (1998), Tamanaha (1996). 
de la richesse, l'efficacité demeure un référentiel implicite. L'appréhension pragmatique du droit continue à se faire en partie sur le mode du calcul. Ce n'est donc pas parce que Posner II ne dit plus ouvertement qu'on évalue les conséquences des règles de droit par rapport à l'efficacité que le critère de l'efficacité - ou une forme différente (" dégradée », du point de vue de Posner I et de la NL\&E) d'efficacité (absorbant par exemple des éléments de rationalité limitée et de subjectivisme judiciaire, le pragmatisme expérimental devenant l'autre nom ou un ersatz de l'efficacité du critère de maximisation de la richesse) - a disparu. L'évolution de Posner de Posner I vers Posner II ne saperait pas tous les travaux antérieurs de Posner I, l'ensemble du NL\&E standard, voire l'idée d'une analyse économique du droit [Harnay et Marciano, 2003].

L'intérêt du pragmatisme juridique de Posner Il est ainsi qu'il reste économique, fondé implicitement sur une logique d'efficacité - même si elle n'est plus forcément celle du critère de maximisation de la richesse. Dès lors, la comparaison entre l'approche de la $N(N) L \& E$ et celle des juges des tribunaux de commerce est heuristique car il y a une dimension économique dans l'activité des tribunaux de commerce, avec un critère sous-jacent d' « efficacité » de leurs décisions, puisque le pragmatisme est vécu par les juges comme adjonction d'économie et de gestion au droit. ${ }^{17}$

Tout en sachant que ce choix est discutable, je prends la responsabilité de cette comparaison en admettant la « nouveauté » de Posner II. Ce passage de Posner I à Posner II nous intéresse ici car il représente une forme d'évolution dans le pragmatisme du juge, évolution qui aboutira à un pragmatisme « expérientiel » très proche de celles des juges consulaires que nous avons approchés au tribunal de commerce de Paris. Le tribunal de commerce français affirme une forme de pragmatisme dans le contrôle social du monde des affaires et des conflits entre entrepreneurs. Nous retrouvons dans son fonctionnement les caractéristiques d'une institution juridique qui généraliserait le pragmatisme de la $N(N) L \& E$. D'une certaine manière, même s'il provient d'un horizon juridique très différent, Posner II retrouve quatre cent cinquante ans d'expérience du juge consulaire français qui cherche à rapprocher le droit des exigences de l'économie et de la gestion, telles qu'elles sont définies par lui(elle)-même en tant que praticien(ne) des affaires. Ces juges consulaires sont des hommes et des femmes d'affaires (non rémunérés, rappelons-le, et parrainés par des syndicats patronaux) qui se prévalent d'un pragmatisme juridique s'appuyant, pour prendre des décisions de justice, sur leurs connaissances de l'économie et de la gestion (connaissances de première main qui feraient défaut, selon eux, aux juges de carrière), mais aussi sur les usages du monde des affaires, leur bon sens et leur sens de l'équité. En théorie, ces magistrats se préoccupent à la fois de droit, d'efficacité (définie par l'économiste et le gestionnaire), des conséquences de leurs décisions et de la satisfaction et de l'adhésion de leur public.

Si le pragmatisme " expérimentaliste » de Posner II est une approche de la décision de justice dont on retrouve beaucoup d'échos chez des représentants de la régulation conjointe des marchés à la française, c'est-à-dire les juges du commerce, alors

\footnotetext{
${ }_{17}$ Pour Harnay et Marciano (2003), l'évolution de Posner de Posner I à Posner Il ne serait peut-être pas si fondamentale ou si radicale. En particulier, il semble que le "Posner mou » (II) constitue en fait une version " hypocrite » du Posner dur (I) de la NL\&E standard. Cela va dans le sens d'une continuité entre Posner l et Posner Il qui resterait à examiner, par les sociologues de l'économie et du droit, dans les pratiques des juges plutôt que dans l'exégèse des textes de la $N L \& E$ ou de la $N(N) L \& E$. Un exemple évident est celui d'une analyse économique du droit de la faillite où le calcul économique (même si différents objectifs peuvent être en jeu) tient clairement une place importante.
} 
le tribunal de commerce français apparaît du même coup comme une institution emblématique à la fois de la régulation conjointe propre à un régime consulaire et de cette approche du pragmatisme juridique « expérientiel » dans le travail du juge de l'économie, pragmatisme qui ne rend pas nécessairement obsolète la valeur d'efficacité. Pour les juges consulaires qui expérimentent depuis quatre cent cinquante ans les principes du $\mathrm{N}(\mathrm{N}) \mathrm{L} \& \mathrm{E}$, les usages sont sources de droit lorsqu'ils sont retenus par le juge expérimentateur qui ne se satisfait pas d'appliquer de manière routinière des décisions de cas précédents ou d'appliquer strictement la législation.

Krecké (2003) voit dans la vision plus modeste de Posner II un gain en réalisme et sens commun, mais aussi une perte de cohérence et de capacité de prédiction de la théorie de la NL\&E. Pour notre part, d'un point de vue de sociologue, le détour par le pragmatisme de Posner II éclaire la principale limite de la théorie pragmatique des juges consulaires, c'est-à-dire sa définition comme purement individuelle. Or, comme le suggérait déjà la présence des usages et des normes sectorielles au TCP, le pragmatisme et l'expérience individuels du juge s'enracinent dans des représentations beaucoup plus collectives. Ainsi, l'examen du raisonnement, des motivations, des carrières et des attributs des juges consulaires [Lazega et Mounier, 2000, 2003, 2007] nous apprend aussi que le juge décide dans un contexte institutionnel qui valorise plus ou moins son raisonnement pragmatique ou certaines dimensions de ce dernier. Là où le juge de la $N(N) L \& E$ est un individu qui tranche seul, le juge que nous avons observé agit dans un contexte de conflits de conventions et de représentations collectives.

Prendre au sérieux l'appareil organisationnel et le cadre institutionnel du travail du juge fait apparaître une nouvelle dimension du pragmatisme, i.e. une dimension collective. On observe plus bas quelques-unes des caractéristiques du contrôle social des marchés qui illustrent l'importance de l'analyse des institutions juridiques, de leurs acteurs collectifs et de leurs formes collectives de pragmatisme. Le rapprochement des attitudes de Posner II et des juges du commerce fait apparaître que le pragmatisme sans théorie des structures organisationnelles et sociales et sans théorie des représentations et conventions est insuffisant pour fonder une théorie convaincante du contrôle social des marchés, en particulier de rendre compte des formes de néo-corporatisme et de capture institutionnelle que l'on observe dans les institutions consulaires.

\section{L'institution pragmatique comme cible d'entrepreneurs judiciaires}

Les tribunaux ne sont pas des institutions statiques qui prennent des décisions atemporelles et purement rationnelles [Heydebrand et Seron, 1990 ; Wheeler et al., 1988]. Ce sont des territoires institutionnels très prisés, qui font l'objet de concurrence et de conflits importants hors tribunal [Flemming, 1998]. Ceci est particulièrement vrai dans le cas des tribunaux où les juges sont eux-mêmes des hommes et des femmes d'affaires parrainés par des syndicats patronaux, sélectionnés pour représenter des secteurs très divers de l'économie et élus par la chambre de commerce de leur juridiction. Dans la mesure où cette régulation conjointe suppose la rencontre entre différents acteurs hétérogènes issus de milieux différents, on peut la comparer à une forme de " gouvernance » des marchés. 
Dans le cas que nous examinons, des intérêts structurés tentent de façonner le tribunal de commerce consulaire, nous considérons les juges tant comme les parties officielles tierces, représentant l'intérêt général, appliquant les règles et les procédures juridiques dans le processus de règlement de conflits, que comme de possibles leviers de surveillance et d'influence non officielles, représentant leur secteur économique d'origine et préconisant potentiellement des solutions à ces conflits, qui ne seront pas préjudiciables à ces intérêts particuliers. En tant que leviers, ils peuvent avoir soit un effet direct sur des décisions judiciaires, soit encore un effet indirect s'ils réussissent à influencer les autres juges qui siègent avec eux. Dans ces conditions, l'une des implications du pragmatisme, tel qu'il est défini par la N(N)L\&E et indépendamment de la cohérence des positions qu'il préconise, est de faire de cette institution de contrôle social des marchés un terrain encore plus contesté qu'à l'accoutumée. Portés par les sensibilités individuelles, les sensibilités collectives et les intérêts corporatistes s'expriment avec légitimité grâce au pragmatisme qui leur sert ainsi de « pied dans la porte ». L'exemple le plus visible de cette stratégie est celui de la domination des banquiers-juristes.

Selon la justification de ce système de régulation conjointe, la sélection des juges devrait produire une représentation très diversifiée des secteurs de l'économie, particulièrement dans les grands tribunaux de commerce comme celui de Paris. Au moment de notre étude, les secteurs représentés par les juges (dans lesquels ils travaillaient ou avaient travaillé) étaient en effet très diversifiés. Ainsi, dans les affaires complexes, les renseignements concernant une spécialité du monde des affaires pouvaient être accessibles au tribunal par le biais de juges issus de cette spécialité. Certains secteurs et/ou entreprises investissent cependant plus que d'autres en entrepreneurship judiciaire et endossent une plus grande partie des coûts du contrôle parce qu'elles ont intérêt à le faire. En théorie tous les syndicats patronaux peuvent présenter des candidats aux élections de juges consulaires sur une base annuelle afin de combler les postes vacants résultant d'un turnover annuel de $10 \%$ du personnel au tribunal. L'analyse montre que, dans les faits, tous ne le font pas. Certains le font plus systématiquement que d'autres ; ainsi, $29 \%$ des juges étaient issus du secteur de la banque / finance. Chaque année, ce secteur présente plusieurs candidats à ces élections ${ }^{18}$.

Le secteur financier est clairement surreprésenté au TCP, en termes absolus et relatifs. En fait, le secteur financier représentait, en 2000, 3 \% de la population active en France ${ }^{19}$ et $5,1 \%$ à Paris où les industries de service sont surreprésentées par comparaison avec la province ${ }^{20}$. En termes de valeur ajoutée à l'économie par branche (sur la base de 1995), la part du secteur des services financiers dans la valeur ajoutée totale à l'économie française était de 5,3\% ${ }^{21}$. Ces indications descriptives suffisent à montrer que les juges issus du secteur financier sont effectivement des leviers possibles de cette industrie et que l'influence qu'ils peuvent exercer sur les autres juges au tribunal représente une menace pour l'indépendance de ce tribunal.

\footnotetext{
${ }^{18}$ Par exemple, en 2003, 21 d'entre eux ont été parrainés par l'Association française des banques et 5 par l'Association française des sociétés financières. Parmi les institutions financières qui employaient des juges actifs (au TCP seulement), BNP-Paribas avait fourni 7 juges, Suez 4, la Société générale 4, le Crédit lyonnais 4, et le Crédit commercial de France 4.

${ }^{19}$ Source : Enquête Emploi 2000, Institut national de la statistique et des études économiques, version CD-ROM.

20 Source : Institut national de la statistique et des études économiques, mensuel n 202, octobre 2001, île-deFrance à la page : «Gros plan sur l'emploi francilien en 1999 ».

${ }^{21}$ Source: Institut national de la statistique et des études économiques, Comptabilité nationale, 2001 (www.insee/ fr/indicateur/cnat_annu/tableaux/t_1201_25_4.htm). En 1998, il y avait 1237 établissements de crédit en France, comptant 25428 caissiers et 714730 employés.
} 
Traditionnellement, cette industrie est un secteur très litigieux [Cheit et Gersen, 2000]. Une part appréciable du rôle des tribunaux de commerce concerne, pour des raisons évidentes, le secteur financier, ce qui motive ses investissements en entrepreneurship judiciaire, par exemple pour limiter ses risques dans les affaires caractérisées par des niveaux de crédit élevés ${ }^{22}$. Un exemple illustre les intérêts du secteur à endosser une part plus grande que la moyenne des coûts du contrôle dans le monde des affaires français : en 1985, le gouvernement socialiste modifiait la loi sur les faillites dans le but de donner la priorité à la lutte contre le chômage et donc aux intérêts des salariés plutôt qu'à ceux des créanciers. La nouvelle loi exige que les juges se prononcent sur la possibilité que l'entreprise survive si elle est mieux gérée [Guéroult et al., 1993]. S'ils décident que l'entreprise - et ses emplois - peuvent être sauvés, ils nomment un administrateur responsable de sa gestion. S'ils décident que l'entreprise ne peut être sauvée, ils ordonnent sa liquidation. Lorsque les juges choisissent un administrateur plutôt qu'un liquidateur, les créanciers doivent attendre et courent donc le risque de perdre encore plus d'argent que si l'entreprise était simplement liquidée. Les banques et les institutions financières sont souvent elles-mêmes des créanciers et risquaient donc, dès la passation de la nouvelle loi, de perdre des sommes énormes. Pendant neuf ans, le secteur financier français a tenté de faire pression sur le personnel politique pour que cette loi soit modifiée ${ }^{23}$. En 1994, une tentative ratée à l'Assemblée nationale semble avoir incité le secteur à changer sa stratégie et à s'employer plutôt à augmenter le nombre de « ses » juges consulaires dans les formations de jugement. En 2006, 21 ans après la loi de 1985, le secteur de la banque parvient à faire modifier le droit des faillites dans un sens qui lui est plus favorable.

\section{Pragmatisme collectif et capture institutionnelle}

Le secteur financier met en jeu de grandes quantités de ressources dans les litiges commerciaux et les faillites. Il est en conséquence disposé à investir dans la redéfinition des règles et il est de son intérêt d'essayer de structurer le tribunal pour imposer ses normes et ses pratiques plutôt que celles d'autres secteurs. Ses priorités (par exemple la conservation d'une valeur élevée des actifs, l'évitement du soutien abusif ou de la rupture abusive de crédit, une grande sensibilité aux répercussions de l'échec des entreprises sur l'économie) peuvent donc être débattues tant du côté du contentieux que de celui des faillites.

Le fait que l'institution pragmatique soit la cible d'entrepreneurs judiciaires crée le risque qu'au pragmatisme « expérimentaliste » de Posner II et de la N(N)L\&E s'ajoute, dans un contexte consulaire de régulation conjointe des marchés, un pragmatisme plus collectif, porteur de normes corporatistes. Pour la N(N)L\&E, l'apprentissage et l'expérience sont nécessairement purement individuels. Or, comme le montrait déjà Durkheim (1898), les représentations individuelles sont structurées par des représentations collectives.

\footnotetext{
${ }^{22}$ C'est ici que le manque de données statistiques désagrégées et accessibles sur les décisions du tribunal de commerce constitue un obstacle à la recherche sur le régime consulaire de contrôle social de l'économie. La Chancellerie ne dispose en effet que de très peu de chiffres sur le travail des juges du tribunal de commerce.

${ }^{23}$ Sur la position de la Conférence générale des tribunaux de commerce sur la loi de 1985, voir Rey (2001), que l'on pourra lire avec la distance procurée par Commons (1924). Une présidente récente du tribunal de commerce de Paris, issue notamment du monde la banque, a exercé une influence déterminante sur la rédaction de la nouvelle loi sur les faillites et la prévention des difficultés des entreprises (2007).
} 
Lorsque les juges partagent leurs expériences et leurs compétences, lorsqu'ils suivent des règles, ils s'appuient sur des fondements construits collectivement. Réduire le problème du jugement pragmatique au raisonnement en équité purement individuel est donc trop limitatif. Lorsque les magistrats consulaires tiennent un discours qui met en avant leur pouvoir souverain d'appréciation et leur devoir moral de juger en s'appuyant sur leur expérience individuelle et leurs connaissances de l'économie et de la gestion, non seulement du droit, les « sensibilités individuelles » prennent le relais de critères et de normes plus collectifs, sectoriels, pour adapter le droit codifié aux réalités toujours en mouvement de l'économie et des affaires. Le règlement efficace des conflits ne peut en effet passer outre tout un ensemble des conventions informelles [Lazega et Favereau, 2002] qui structurent de manière variable les pratiques du monde des affaires. On retrouve, sous les sensibilités individuelles, des " sensibilités collectives », c'est-à-dire des conventions partagées [Lazega et Mounier, 2008b]. Ces sensibilités caractérisent non pas simplement des individus isolés mais l'ensemble des individus provenant d'un secteur spécifique de l'économie.

Cette régulation conjointe apparaît ainsi comme le lieu d'une lutte d'influence pour la construction d'un cadre de référence commun indispensable à la qualification et à l'interprétation stabilisée des faits. Dans cette concurrence « épistémique » entre secteurs, celui de la banque et de la finance (même s'il ne s'est pas toujours formellement organisé pour cela) est en position de promouvoir ses sensibilités et représentations collectives, des critères de justice et des conventions bien identifiables.

La recherche empirique montre qu'il existe une relation entre la carrière, l'appartenance sectorielle et la sensibilité que les juges consulaires mobilisent dans des affaires où ils disposent d'un pouvoir souverain d'appréciation. Par exemple, les juges à la fois juristes et issus du monde de la banque et de la finance tendent à adopter une attitude plus interventionniste que l'ensemble des juges dans les litiges opposant les actionnaires d'une entreprise, en intra-organisationnel [Lazega et Mounier, 2007, 2008a] ; en cas de litige entre actionnaires, ils sont plus enclins à privilégier l'intérêt social d'une société (tel que défini par la direction et donc l'actionnaire majoritaire) que les autres répondants, et moins hostiles à l'intervention du juge dans la vie d'une société. Ils ont en revanche une attitude beaucoup moins interventionniste que l'ensemble des juges dans les litiges opposant les parties d'un contrat, sur un marché, en inter-organisationnel. Ils ont enfin une attitude moins « punitive » que l'ensemble des autres juges consulaires à l'égard de préjudices qualifiés de « moraux » et résultant de comportements de concurrence déloyale perturbant le fonctionnement (supposé naturel) des marchés.

C'est l'inverse qui est observé pour les juges issus du monde du BTP : ils ont tendance à adopter une attitude moins interventionniste que l'ensemble des juges dans le litige opposant les actionnaires d'une entreprise, en intra-organisationnel. Ils ont en revanche une attitude beaucoup plus interventionniste que l'ensemble des juges dans le litige opposant les parties d'un contrat sur un marché, en inter-organisationnel. Ce contraste est confirmé par l'attitude moins punitive adoptée par les banquiers-juristes que par les juges issus du BTP à l'égard d'une demande recourant au préjudice moral d'une personne morale dans le fonctionnement des marchés. Rappelons aussi que les juges les plus jeunes ont tendance à être plus interventionnistes et punitifs que les plus anciens à la fois dans les marchés et dans les entreprises. 
En envoyant systématiquement au tribunal de commerce des cadres supérieurs qui ont une formation juridique, la banque / finance domine cognitivement le tribunal. Parmi les sensibilités à la fois individuelles et collectives, celles des juges venant des secteurs de la banque et de la finance et ayant un diplôme en droit (banquiers-juristes) attirent particulièrement l'attention parce qu'ils sont les juges les plus centraux dans le partage des compétences et de l'expérience caractérisant la communauté des juges du TCP pendant les années où nous avons pu l'approcher. Nous avons en effet observé cette influence en examinant les échanges de conseils entre les juges, échanges que nous présumons servir à poser les prémisses des décisions judiciaires. Le réseau de conseil parmi les juges et son efficacité dans l'établissement des prémisses des décisions sont considérés comme une articulation forte entre structure de l'organisation et rationalité des membres [Lazega, 1992, 2001 ; Lazega et al., 2006, 2008]. En effet, les régularités observées dans les échanges de conseils dans le tribunal montrent « qui est prêt à écouter qui » au moment où il faut définir les problèmes et prendre une décision judiciaire.

Cette domination par les juges juristes issus de la banque n'est pas pour autant une domination nécessairement confortable. L'intégration sociale des banquiers au tribunal est relativement plus faible que celle des juges issus d'autres secteurs, précisément parce que le secteur bancaire a un si grand nombre de représentants. Avoir une forte présence en termes de " position » (Flemming) peut se révéler parfois contreproductif en termes d'influence. Le secteur financier est perçu par les juges provenant des autres secteurs soit comme défenseur de ses intérêts corporatistes soit comme essayant de se substituer à l'État, une fois que ce dernier s'est retiré du contrôle direct de l'économie. C'est ce qu'un juge, lui-même ancien banquier, exprime par cette formule : «Les commerçants détestent la banque ». Notons, comme autre indicateur de cette tension entre les représentants de la finance et les autres, le fait que les juges issus du secteur financier sont presque systématiquement ceux qui ont été élus avec le plus petit nombre de voix ${ }^{24}$.

\section{Repenser l'action publique à partir d'une critique du régime consulaire}

Notre intérêt pour le tribunal de commerce de Paris comme institution consulaire vient de ce qu'elle représente une institution de régulation conjointe des marchés, conjointe au sens où elle articule les efforts de l'État (il s'agit d'une institution judiciaire), du monde des affaires et de ses représentants (à la chambre de commerce) et de la société civile. Caractéristique de ce régime consulaire, depuis plus de quatre siècles, l'expérience du monde des affaires et la connaissance de l'économie ont constitué un argument fort en faveur d'une justice commerciale mise en œuvre par des juges consulaires. La complexité des tâches accomplies par les juges du commerce, aussi bien dans le domaine du contentieux général ou spécialisé que dans celui des procédures collectives, renvoie à l'importance des compétences et expériences

\footnotetext{
${ }^{24}$ Le juge élu avec le moins de voix porte le nom de "culot »; jusqu'à très récemment, le culot subissait pendant sa première année des brimades rituelles parmi ses pairs (porter la serviette de son président de Chambre, se taire à table au déjeuner hebdomadaire de sa Chambre, etc.). Les culots des promotions récentes étant toujours des banquiers, ce détail ethnographique donne une idée de la manière dont les non-banquiers perçoivent les banquiers.
} 
multiples (juridiques, économiques, gestionnaires, entre autres) mobilisées dans ce type d'institution.

Les juges consulaires disposent en effet de toutes sortes de moyens pour chercher des informations et des conseils sur lesquels appuyer leurs raisonnements et leurs décisions. Mais l'un des arguments forts par lesquels de nombreux juges de grands tribunaux de commerce justifient le fonctionnement consulaire traditionnel de la justice commerciale est que des compétences très diverses sont représentées au sein de ces institutions. Les juges sont donc en mesure de puiser dans l'expérience de leurs collègues de spécialités différentes, provenant de milieux hétérogènes. Au sein du tribunal, ils sont identifiés par leurs compétences pour que l'on sache qui consulter sur quoi. La connaissance est ainsi « distribuée ». Ceci rend systématique l'examen empirique de l'organisation informelle de la consultation (hors délibéré) au tribunal de commerce de Paris. II s'ensuit assez « naturellement » que l'institution consulaire s'appuie sur cette expérience et valorise le pragmatisme pour légitimer son existence.

En résumé, l'étude du fonctionnement d'une institution consulaire et pragmatique de régulation conjointe des marchés, le tribunal de commerce de Paris, fait apparaître la proximité entre les thèses de la New (New) Law \& Economics sur le « pragmatisme expérientiel » ou le « pragmatisme quotidien » avec la pratique du jugement en équité des juges du commerce qui s'appuient sur leur connaissance de l'économie et la gestion pour prendre des décisions en matière de contentieux économique et de faillites. La mise en évidence du contrôle de cette institution pragmatique par le monde (et les critères) de la banque/finance expose cependant l'existence, sousjacente au pragmatisme " individuel » enraciné dans l'expérience quotidienne du juge, d'un pragmatisme « collectif » porteur de valeurs et d'intérêts néo-corporatistes. Cette analyse du pragmatisme juridique mis en avant par les juges de ce tribunal permet de caractériser le régime consulaire de contrôle social des marchés. Ce constat soulève la question de la protection de l'intérêt général [Lazega, 2003], de la mise en œuvre du pragmatisme juridique et de la perspective de la N(N)L\&E dans les institutions de plus en plus nombreuses auxquelles l'État délègue ses pouvoirs.

En effet, le pragmatisme, tel qu'il est présenté par cette institution et par la N(N)L\&E, est un pragmatisme individuel. Or, à l'échelle d'une institution consulaire comme le tribunal dont nous avons examiné le fonctionnement, le pragmatisme acquiert une dimension politique. Du fait de l'identité des juges, du fait de leur mode de recrutement et d'apprentissage, du fait de l'existence d'intérêts, d'usages et de représentations collectives caractéristiques des métiers et des industries spécifiques d'où proviennent ces juges, du fait des processus de résolution des divergences et controverses normatives au sein de l'institution, le pragmatisme est porteur d'une forme de néo-corporatisme dont l'existence et le pouvoir menacent l'impartialité du tribunal.

Le néo-corporatisme ne se réduit certes pas à la promotion d'une forme de pragmatisme collectif. La construction sociale de l'« irréversibilité » du design institutionnel du TCP en témoigne. Une fois le pragmatisme collectif institué, une fois qu'il a ouvert la porte aux influences exogènes des normes sectorielles, il est plus difficile de séparer le jugement pragmatique d'un juge individuel des intérêts corporatistes dont il est porteur. Reste, on l'a noté plus haut, que cette institution de régulation conjointe des 
marchés, où le monde des affaires participe directement au fonctionnement de l'appareil d'État, est d'une longévité étonnante. Le pragmatisme individuel de ses juges et son ouverture aux néo-corporatismes des industries qui cherchent à y exercer une influence ne suffisent évidemment pas à expliquer cette longévité. L'observation du fonctionnement de cette institution et de ses relations avec son environnement montre qu'elle rend des services aux pouvoirs publics (les magistrats consulaires ne sont pas rémunérés ; ils s'occupent des faillites ${ }^{25}$ sans compromettre le personnel politique en cas de licenciements massifs; ils forment les jeunes magistrats issus de l'Ecole nationale de la magistrature aux arcanes du monde des affaires), qu'elle est soutenue par un système inter-organisationnel fortement intégré (auquel appartiennent la chambre de commerce et d'industrie et les syndicats patronaux), que la surveillance statistique dont elle fait l'objet de la part de la Chancellerie est très superficielle (ce qui lui permet, à elle et à son administration -le greffe -, d'entretenir une certaine opacité sur ses décisions et sa gestion des conflits d'intérêts).

De plus l'institution est soutenue par un milieu social qui s'est construit autour d'elle : les juges consulaires sont les principaux « recruteurs » de nouveaux juges; ils sont aussi des amis du candidat ou des membres de sa famille [Falconi et al., 2005]. Enfin, le pragmatisme juridique s'accompagne d'une rhétorique de profession libérale qui s'avère très utile pour construire l'affichage de l'indépendance du juge et de l'institution consulaire (insistance sur la complexité des tâches et la gestion de l'incertitude, élitisme dans la sélection avant l'élection-cooptation, affirmation du service public désintéressé, partage collégial des compétences, organisation de la conférence des juges consulaires de France comme organisation de représentation et de contrôle de ses membres qui, pour les juges consulaires, remplit des fonctions pratiques et symboliques presque similaires à celle d'un Ordre -sans pour autant être une profession).

Dans le contexte anglo-saxon, où l'apparence d'un conflit d'intérêts est aussi grave qu'un conflit d'intérêts, cette forme de capture institutionnelle peut éventuellement être évitée. Dans une culture juridique où l'apparence d'un conflit d'intérêt n'est pas prise au sérieux, ce néo-corporatisme pose un problème politique. Le modèle consulaire et le pragmatisme sont porteurs d'une option politique qui n'est pas discutée sur la place publique. Dans ce régime de contrôle social des marchés, une partie de l'administration de l'État, et donc du gouvernement, fonctionne de manière pragmatique, s'auto-perpétuant de manière néo-corporatiste et élitiste. L'existence de l'institution elle-même devient difficilement compatible avec l'article 6 de la Convention européenne des droits de l'Homme et sa définition de l'impartialité.

La forme consulaire de la régulation conjointe des marchés représente une prise de contrôle d'une partie de l'appareil d'État qui dépasse la privatisation des services publics. En ce sens, les questions qu'elles posent ajoutent encore de nouvelles questions à celles que posaient la sociologie politique ou la science politique des années 1980 [Chevallier, 1978 ; Segrestin, 1985 ; Jobert et Muller, 1987]. La forme consulaire d'action publique se diffuse aujourd'hui, par exemple dans le fonctionnement de

\footnotetext{
${ }^{25}$ D'une certaine manière, les juges consulaires acceptent de faire une part du « sale boulot » à la fois des pouvoirs publics et de l'économie de marché. En mettant au service de l'intérêt général leur expertise et leur expérience, ils posent au sociologue d'une manière très spécifique la question plus générale d'Everett Hugues (1962) : celle des "Good people and dirty work».
} 
l'arbitrage ou dans celui de l'État « régulateur » qui promeut des autorités administratives indépendantes pour exercer des fonctions appartenant à son cœur de métier. Notre travail suggère que le champ de la sociologie économique pourrait bénéficier plus systématiquement de recherches organisationnelles et structurales, notamment en s'intéressant davantage à des institutions économiques de ce type, à l'équilibre complexe de régulation conjointe qu'elles préconisent, aux formes complexes de privatisation de l'État qu'elles représentent et, finalement, aux gagnants et aux perdants de chacun de ces équilibres.

Enfin, pour en revenir au domaine académique, ces constats impliquent que la privatisation de fonctions étatiques n'est pas une désinstitutionnalisation, mais le remplacement progressif d'institutions statutaires par des institutions dont le fonctionnement s'inspire du régime consulaire. II convient dès lors de repenser l'action publique à partir d'une critique du régime consulaire plutôt qu'à partir d'une sociologie des groupes professionnels [Le Bianic et Vion, 2008]. Par ailleurs, I'attention des sociologues de l'économie et du droit aux normes environnantes d'institutions comme le tribunal de commerce est aujourd'hui beaucoup plus forte que celle de l'économie du droit en général [Lazega, 2003 ; Swedberg, 2003]. II faut cependant mentionner, toujours sur un plan très général, l'existence d'un questionnement commun autour des notions d'autorégulation, de co-régulation et, peut-être, de régulation conjointe avec l'économie des institutions, des conventions et de la régulation. II existe des parallèles entre l'approche que nous avons mis en œuvre du pragmatisme collectif et de la privatisation de l'État avec les analyses économiques de l'école des choix publics et de la recherche de rente. Resterait à faire apparaître des hypothèses et méthodologies communes à la sociologie économique du droit et ces approches économiques. Dans ce domaine, tout ou presque reste à faire.

\section{Références bibliographiques}

Ayres, I. et J. BratThwalte (1992), Responsive Regulation: Transcending the Deregulation Debate, Oxford University Press, Oxford.

Brint, M. et W. Weaver (1991), Pragmatism in Law and Society, Westview Press, Boulder, Co.

Carruthers, B.G. et T. Halliday (1998), Rescuing Business: The Making of Corporate Bankruptcy Law in England and the United States, Oxford, Oxford University Press.

CHAPUT, Y. (2002), "L'indépendance renforcée du juge économique », Revue de Jurisprudence Commerciale, Numéro spécial Colloque de La Baule : Le Juge de l'économie, 46, p. 74-94.

Chetr, R.E. et J.E. Gersen (2000), « When Businesses Sue Each Other: An Empirical Study of State Court Litigation », Law \& Social Inquiry, vol. 25, p. 789-816.

ChevalueR, J. (1978), Variations autour de l'idéologie de l'intérêt général, PUF, Paris.

CoAsE, R. H. (1960), « The Problem of social cost», Journal of Law and Economics, 3, p. 1-44

Commons, J.R., (1924). Legal Foundation of Capitalism, New-York, McMillan.

Coutant, M.-F., Les Tribunaux de commerce, Paris, PUF, coll. « Que sais-je ? ».

Dicksteln, M. (1998), The Revival of Pragmatism: New Essays on Social Thought, Law, and Culture, Durham, Duke University Press. 
DuRKнelм, É. (1898), "Représentations individuelles et représentations collectives », Revue de Métaphysique et de Morale, tome $\mathrm{VI}$,

Falconi, A. M., K. Guenfoud, E. Lazega, C. Lemercier, L. Mounier (2005), « Le contrôle social du monde des affaires: une étude institutionnelle », L'Année sociologique, 55(2), p. 451-484.

FarbeR, D. (1995), «Reinventing Brandeis: Legal Pragmatism for the Twenty-First Century ", University of Illinois Law Review, 163.

Favereau, O. (1994), « Règles, organisation et apprentissage collectif : un paradigme non standard pour trois théories hétérodoxes », dans A. Orléans (dir.), Analyse économique des conventions, PUF, Paris.

FavereAu, O.and Lazega, E.(eds.) (2002), Conventionsand StructuresinEconomicOrganization: Markets, Networks, and Hierarchies. Edward Elgar Publishers, Cheltenham.

Flemming, R.B. (1998), " Contested Terrains and Regime Politics: Thinking about America's Trial Courts and Institutional Change », Law \& Social Inquiry, vol. 23, p. 941-965.

Grabosky P. et Bratthwaite J. (1986), Of Manners Gentle, Enforcement Strategies of Australian Business Regulatory Agencies, OUP, Oxford.

Grey, T. G. (1996), « Freestanding Legal Pragmatism », Cardozo Law Review, 18, p. 21-42.

Grey, T. G. (1989), « Holmes and Legal Pragmatism », Stanford Law Review, 41, p. 787-870.

Guéroult, A., H. Lamotte et B. du Marals (1993), Les garanties et le crédit aux entreprises, Rapport du Conseil national du crédit, Paris, Éditions de la Banque de France.

HaehL, J.-P., Hamon, B. et B. Munoz-Perez (1992), « Le contentieux général devant les tribunaux de commerce en $1990 »$, Infostat Justice, $n^{\circ} 31$.

Haehl, J.-P., Hamon, B. et B. Munoz-Perez (1993), « Le contentieux général devant les tribunaux de commerce en $1991 »$, Infostat Justice, $n^{\circ} 33$.

Harnay, S. et Marciano, A. (2003), Posner et l'analyse économique du droit. Éditions Michalon, coll. «Le bien public ».

Hawkins, Keith O. et Thomas, J.M. (eds.) (1984), Enforcing Regulation, Kluwer-Nijhof, Den Haag.

Heydebrand, W. et C. Seron (1990), Rationalizing Justice: The Political Economy of Federal District Courts, Albany, SUNY Press.

HILAIRE, J. (1998), « Grandeur ou servitude de la justice consulaire ; la controverse de l'équité ", Justices, $\mathrm{n}^{\circ} 1$.

HILARE J. (1999), « Perspectives historiques de l'élection du juge consulaire », J. Krynen (éd.), Lélection des juges. Étude historique française et contemporaine, Paris, PUF, coll. "Droit et Justice ».

HIRSCH, J.-P. (1985), Les deux rêves du commerce. Entreprise et institution dans la région lilloise (1780-1860), Éditions de l'EHESS, Paris.

Hughes, E. C. (1962), « Good People and Dirty Work », Social Problems, 10, p. 3-11.

IthuRBIDE, R. (1970), Histoire critique des tribunaux de commerce, LGDJ, Paris.

JOBERT, B. et MuLLER, Pierre (1987), L'État en action. Politiques publiques et corporatismes, PUF, Paris.

KRECKÉ, É. (2003) «Economic Analysis and Legal Pragmatism », International Review of Law and Economics, 23, p. 421-37. 
KreCKÉ, É. et KreCKÉ, C. (2006), "The anti-foundational dilemma: Normative implications for the economic analysis of law », in Krecké, E., Krecké, C. and Koppl, R. (eds.), Cognition and Economics, Collection Advances in Austrian Economics, Volume 9, Elsevier, New York.

Kronman, A.T. (1980) «Wealth Maximization as a Normative Principle », Journal of Legal Studies 9(2), p. 227-242.

Lafon, J. (1981), Juges et consuls à la recherche d'un statut dans la France d'Ancien Régime, Paris, Economica.

LAZEGA, E. (2003), « Networks in legal organizations: On the protection of public interest in joint regulation of markets», Wiarda Chair Inaugural Address, Wiarda Institute Publications, Faculty of Law, Utrecht University.

Lazega, E. et Favereau, O. (2002), «Introduction », in Olivier Favereau et Emmanuel Lazega (eds), Conventions and Structures in Economic Organization: Markets, Networks, and Hierarchies, Edward Elgar Publishing, Cheltenham, p. 1-28.

LAZEGA E. et MOUNier L. (2001), Approche organisationnelle et structurale d'un tribunal de commerce, une étude de faisabilité, Rapport à la mission de recherche "Droit et Justice », ministère de la Justice, septembre 2001.

LAZEgA, E. et Mounier, L. (2002), « Interdependent Entrepreneurs and the Social Discipline of their Cooperation: Structural Economic Sociology for a Society of Organizations», in O. Favereau et E. Lazega (eds.), Conventions and Structures in Economic Organization: Markets, Networks, and Hierarchies, Edward Elgar Publishers, Cheltenham.

LazegA, E. et Mounier, L. (2003a), "Interlocking Judges: On Joint External and SelfGovernance of Markets », in V. Buskens, W. Raub et C. Snijders (eds), Research in the Sociology of Organizations, 20, p. 267-296.

LAZEGA, E. et Mounier, L. (2003b), " Lorsque l'État se retire du contrôle de l'économie, qui prend le relais ? ", in Jean Crête (ed.), Hommage à Vincent Lemieux : La science politique au Québec, le dernier des maîtres fondateurs, Presses de I'Université Laval, p. 155-78.

Lazega, E. et L. Mounier (2003c), « La Régulation conjointe des marchés : Le cas du tribunal de commerce de Paris », in B. Convert (dir.), Repenser le marché, Paris, L'Harmattan, Cahiers Lillois d'Economie et de Sociologie, $\mathrm{n}^{\circ} 41$.

Lazega E. et L. Mounier et al. (2003), Régulation conjointe et partage des compétences entre les juges du tribunal de commerce de Paris, Rapport à la mission de recherche «Droit et Justice », Ministère de la Justice.

Lazega E. et L. Mounier avec la collaboration de A.M. Falconi (2007), Partage des compétences entre magistrats consulaires et usages du monde des affaires au Tribunal de Commerce de Paris, mission de recherche « Droit \& Justice », ministère de la Justice.

LAZEGA, E. et Mounier, L. (2008a), «Quête de statut social, partage des compétences et néo-corporatisme au Tribunal de Commerce de Paris », in Hélène Michel et Laurent Willemez (eds), La justice au risque du profane, Paris, PUF, collection CURAPP.

LAZEGA, E. et L. Mounier (à paraître 2008b), « La rhétorique des professions libérales au service de la privatisation de l'État : le cas des juges consulaires du Tribunal de Commerce français », in D. Demazière et C. Gadea (eds), Sociologie des groupes professionnels : Acquis récents et nouveaux défis, La Découverte, coll. « Recherches ». 
LAZEgA, E. et Mounier, L. (À PARAÎTREXXXXXXX 2008c), " Polynormativité et contrôle social du monde des affaires : le cas de l'interventionnisme et de la punitivité des juges du Tribunal de Commerce de Paris », Droit et Société.

LAZEGA, E., Lemercier, C. et Mounier L. (2006), « A spinning top model of formal structure and informal behaviour: Dynamics of advice networks in a commercial court », European Management Review, 3, p. 113-122.

Lazega, E., L. Mounier, T. Snijders et P. Tubaro (2008), « Réseaux et controverses : De l'effet des normes sur la dynamique des structures », Revue française de sociologie, 49, p. 467-498.

LE BIANIC, T. et VION, A. (2008), Action publique et légitimités professionnelles, Paris, LGDJ.

LemercIer, C. (2003), Un si discret pouvoir. Aux origines de la chambre de commerce de Paris, 1803-1853, La Découverte, Paris.

McINTOSH, W.V. et C.L. CATEs (1997), Judicial Entrepreneurship: The Role of the Judge in the Marketplace of Ideas, Greenwood Press, Westport, CT.

MerCuRo, N. et Medema, S. G. (2006). Economics and the law: From Posner to post-modernism and beyond, Princeton University Press, Princeton.

Montebourg, A. et Colcombet, F. (1998), Les tribunaux de commerce: Une justice en faillite?, Rapport $n^{\circ} 1038$, Commission d'enquête parlementaire de l'assemblée nationale, Paris, Michel Lafon.

Ogus, A. (2006), Costs and Cautionary Tales - Economic Insights for the Law, Hart Publishing, Oxford.

Posner, R. A. (1977), Economic Analysis of Law; Little, Boston and Toronto, Brown and Company, $6^{\text {th }}$ ed., 2003.

Posner, R. A. (1980), «The Ethical and Political Basis of the Efficiency Norm in Common Law Adjudication »; Hofstra Law Review 8(3), p. 487-507.

Posner, R. A. (1993), The Problems of Jurisprudence, Harvard University Press, Cambridge, Mass.

Posner, R.A. (1995), Overcoming Law, Harvard University Press, Cambridge, Mass.

RADIN, M. J. (1990), «The Pragmatist and the Feminist», Southern California Law Review, 631699, p. 1707-1709.

REY, P. (2001), La Réforme de la loi de 1985 sur le traitement des difficultés des entreprises. Rapport au congrès national des tribunaux de commerce, Lyon, le 23 novembre 2001. Publication de la Conférence générale des tribunaux de commerce.

Reynaud, J.-D. (1989), Les règles du jeu, Armand Colin, Paris.

SEGRESTIN, D. (1985), Le Phénomène corporatiste, Fayard, Paris.

SunsteIn, R. C. (2000) (ed.) Behavioral Law and Economics, Cambridge University Press, Cambridge, Mass.

SWEDBERG, R. (1993), «Economics and Custom », Journal of Institutional and Theoretical Economics, 149, p. 204-209.

SwedberG, R. (2003), «The Case for an Economic Sociology of Law », Theory and Society 32, p. $1-37$

TamanaHA, B. Z. (1996), « Pragmatism in U.S. Legal Theory: Its Application to Normative Jurisprudence, Sociolegal Studies, and the Fact-Value Distinction », American Journal of Jurisprudence, 41.

Wheeler, S., K. Mann, A. Sarat (1988), Sitting in Judgment: The Sentencing of White Collar Criminals, New Haven, Yale University Press. 